\title{
Treating Infant Population Fracture Mandible by Prof. Katpar's Innovative Splint technique: Reported from Pakistan
}

\author{
Shahjahan Katpar ${ }^{1 *}$, Maria Shabbir ${ }^{2}$, Muhtada Ahmed ${ }^{3}$, Jawad Safdar ${ }^{3}$, \\ Shoaib Ayub ${ }^{4}$ and Muneer Zardari ${ }^{5}$ \\ ${ }^{1}$ Dean, Faculty of Dentistry and Head Department of Oral and Maxillofacial Surgery, \\ Dow Dental College, Dow University of Health Sciences, Karachi, Pakistan \\ ${ }^{2}$ Department of Oral and Maxillofacial Surgery, Shaheed Mohtarma Benazir Bhutto \\ Institute of Trauma and Former Lecturer Dow Dental College, DUHS, Karachi, Pakistan \\ ${ }^{3}$ Assistant Professors, Department of Oral and Maxillofacial Surgery, Dow Dental \\ College/DUHS, Karachi, Pakistan \\ ${ }^{4}$ Former Lecturer, Department of Oral and Maxillofacial Surgery, Dow Dental College/ \\ DUHS, Karachi, Pakistan \\ ${ }^{5}$ Lecturer, Department of Oral and Maxillofacial Surgery, Dow Dental College/DUHS, \\ Karachi, Pakistan \\ *Corresponding Author: Shahjahan Katpar, Dean, Faculty of Dentistry and Head Department \\ of Oral and Maxillofacial Surgery, Dow Dental College, Dow University of Health Sciences, \\ Karachi, Pakistan. E-mail: profkatpar@duhs.edu.pk, shahji_katpar@hotmail.com
}

\begin{abstract}
Introduction: Mandibular fractures occur relatively less frequent in infantile population as compared to adults, due to elasticity of bone, protected anatomical structures and infrequent exposures to road traffic accidents etc. The treatment procedures involved for such cases is different from those of adults, due to concerns regarding growth, developing Dentition and early healing.

Case Report: Here we discuss case of 8 months old female infant patient presented with fracture of anterior mandible following fall from stairs. The fractured jaw was reduced at theatre without any impression taking and cast formation procedure for acrylic splint and we avoiding using resorbable bone plates. Instead, we used Compound impression material cake piece which is a muco-compressive, thermoplastic material, used as a substitute for an acrylic splint. This was secured with silk suture and rubber tube pieces, replacing circum-mandibular stainless steel wire with silk following manual closed reduction of fracture, under general anesthesia. Results: The clinical application of this technique results in timely and adequate reduction of fracture in pediatric population which was confirmed on post-operative radiographs and clinical follow-up visits of patient and their family reviews.

Conclusion: The article provides an innovative, pioneer, non-invasive surgical procedure to treat infantile mandibular fracture cases by "Prof. Katpar's DUHS splinting technique" within early days of trauma, avoiding costly resorbable bone plates, acrylic splints and early reduction.

Keywords: Acrylic Splint; Prof. Katpar's Splint Technique; Circum-Mandibular Silk Thread; Closed Reduction; Impression Compound; Pediatric Fracture Mandible; Resorbable Bone Plates; DUHS; Pakistan
\end{abstract}

\section{Abbreviation}

DUHS: Dow University of Health Sciences

\section{Introduction}

A pediatric mandibular fracture can cause a child severe pain and the parent or caregiver extreme worry [1]. While the pattern of fractures in infants and children is similar to adults, however, due to a number of factors, including the anatomical complexity of the developing mandible, the management of such fractures differs from those of adults and can greatly challenge the pediatric maxillofacial surgeon [1,2]. Mandibular fractures are the most common seen (56\%) facial skeletal injury in hospitalized pediatric trauma patients [3]. Treatment principles of pediatric mandibular fractures differ from treatment of the adult population in that, a conservative approach is favored and advocated in most cases [4]. This is not only due to different consequences of trauma, but also due to timings and management techniques used. These in turn 
should also be modified to address the child's particular stage of anatomic, physiologic and psychological development [5]. The Oral and Maxillofacial Surgeons must constantly weigh the risks of exploratory open surgical intervention for pediatric mandible fractures against closed procedures and all attempts be made to provide, as early treatment as possible. This in turn is associated with the wonderful rapid healing capacity of children, as delayed treatment leads to numerous form and functional issues. The majority of pediatric mandibular fractures can be managed with closed techniques using short periods of maxillomandibular fixation, acrylic splints or training elastics alone etc. In our community availability of resorbable bone plates is very rare and almost non-existence at all in tertiary care Public sector centers due to its high cost and associated lack of hospital resources and this also increase the stress to provide early treatment for all concerned [6].

We have searched literature and do not find any mandibular fracture case treated via Prof. katpar's DUHS Splinting technique for being: pioneer, non-invasive, innovative and cost effective being reported.

\section{Case Report}

An eight months old female child, not known to have any medical illness, presented to oral and maxillofacial outpatient clinic with chief complaints of mild painful swelling at chin region and difficulty during feeding/eating after falling from stairs. Detailed history revealed that she sustained some injury in her lower jaw after a fall, playing 3 days ago. There was no history of loss of consciousness, convulsion, or vomiting and she was conscious and well oriented. Clinical evaluation revealed minimum extra oral tender swelling over the chin and slight asymmetry on right side of mandible. Intraoral examination revealed step deformity on right side of mandible with small, soft callus on the lingual side of fracture mandible, having erupted deciduous central incisor (Figure 1a). A 3D CT scan revealed mild-moderate displaced, overriding fracture symphysis mandible (Figure 1b), without any other associated fracture of facial skeleton.

Following baseline investigations and nasal intubation under aseptic measures fracture mandible (Figure 1c) was reduced as closed reduction via careful manipulation. Mild Lingual mandibular step deformity was corrected, dental impression compound material of about 2 inches cake piece was immersed in mild hot water and adapted over occlusal surface of mandible after reduction extending in sulcus region (Figure 1d). The 0-0 silk suture was used in a straight needle, as standard circummandibular silk suture technique, replacing SS wire and tied over
3 points to mandible to secure compound splint (Figure 1e) preadapted compo splint is then secured with silk suture (Figure 1f).

Endotracheal tube; size 5 was selected according to external surface, lower jaw of mandible, made small and placed over chin from angle to angle region extra-orally, acting as extra-oral splint (Figure 1g). The fractured mandible was sandwiched via splint, avoiding IMF intra orally with compo splint and extra orally with ETT (Endo tracheal Tube) piece for 7 - 10 days.

Postoperative recovery was uneventful. On 1st day I/V antibiotics were given and next day patient was discharged with oral medications for 2 more days and parents were instructed to be vigilant regarding displacement of compo material into throat or airway in which situation immediately rush to hospital. Composplint was kept in place and removed on 7 post op day following PA view face, showing no obvious sign of displacement (Figure $1 \mathrm{~h}$ ). No clinical mobility was present at the fracture site following removal of Compound splint. Patient was kept on weekly follow up for up to one month, then after regular 3 months (Figure 1i). Till date on follow up, patient has no reduction in mouth opening or deviation of mandible, feeds well and we see a happy family.

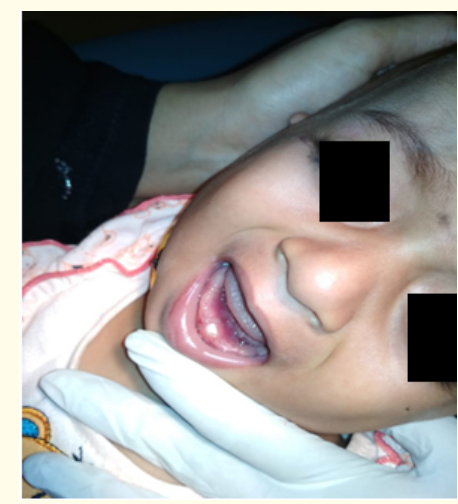

Figure 1a: Pre-op clinical picture.

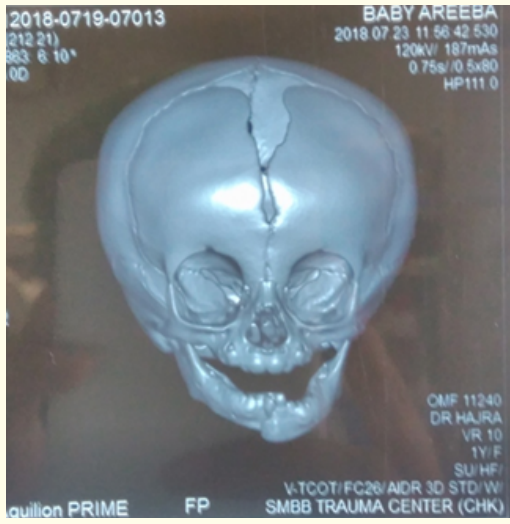

Figure 1b: Preoperative 3D Scan. 


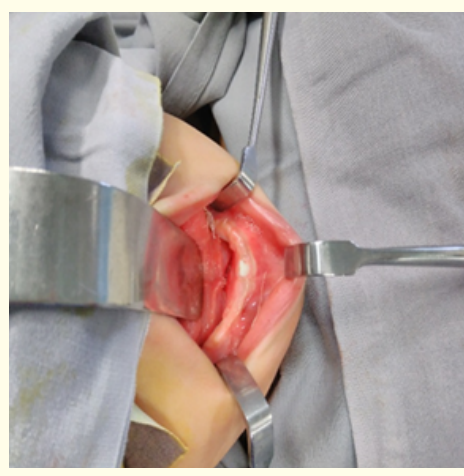

Figure 1c: Intra-op presentation.

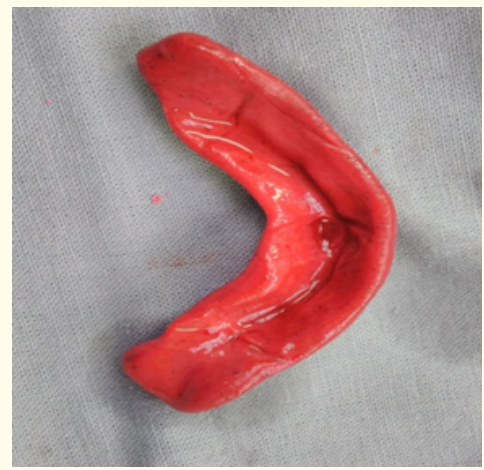

Figure 1d: Compo-splint with central incisor tooth imprint.

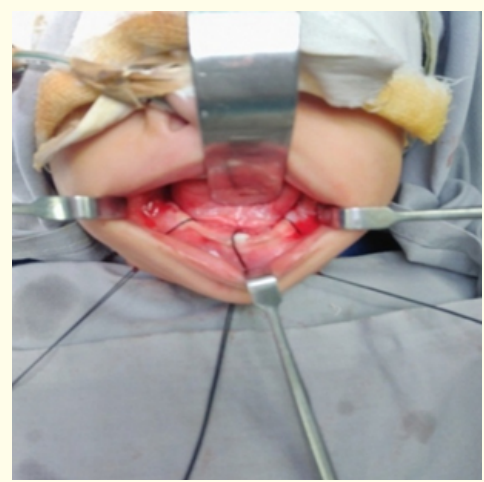

Figure 1e: Circum-mandibular silk suture.

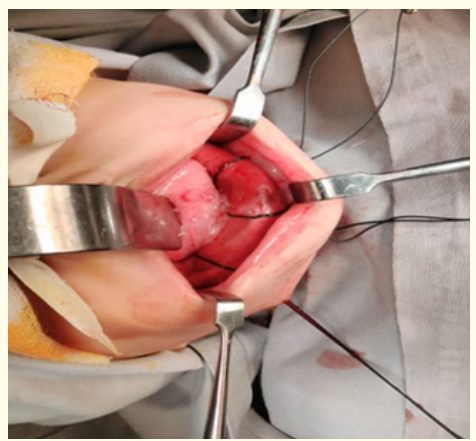

Figure 1f: Silk suture over compo-splint.

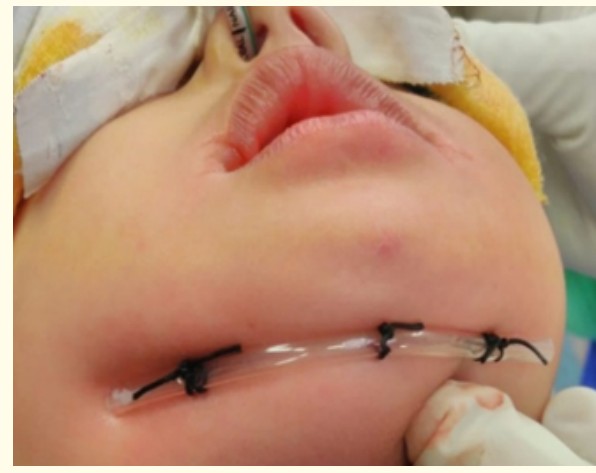

Figure 1g: External ETT splint.

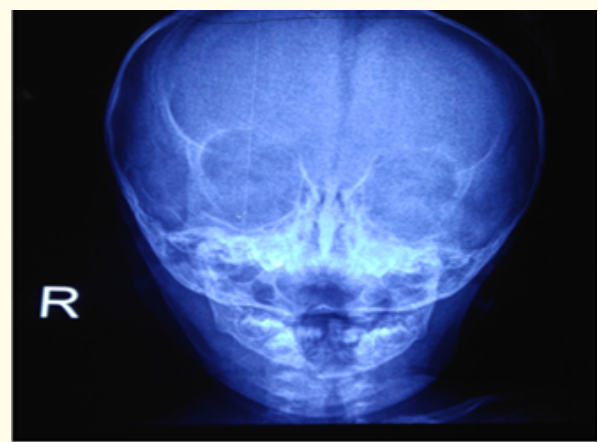

Figure 1h: Post op PA view.

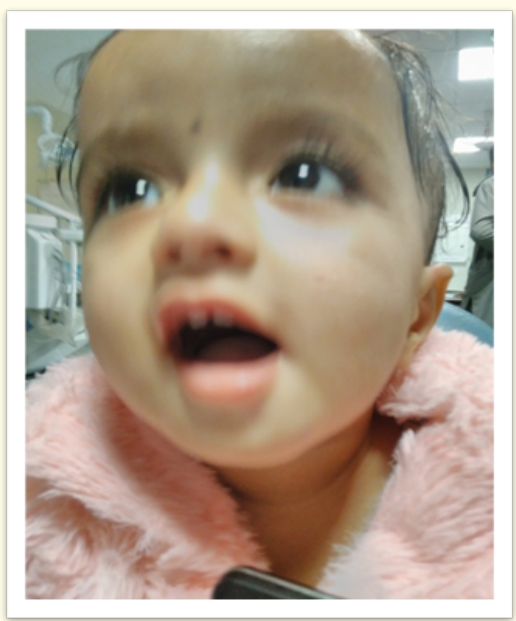

Figure 1i: 3 months follow up.

\section{Discussion}

The fractured pediatric mandible does not need high degree of precision for reduction, as its precision is insignificant, due to rapid osteogenic potential for bony repair, absence of erupted teeth the minor imperfections remodel. Prompt management is essential to achieve an optimal outcome as bony fragments become partially united in 4 - 6 days making fractures difficult to reduce by the 
7 th day onwards. Thus, timing of surgical intervention via this procedure reported must preferably be done within the first 72 hours for maximum benefits. Although individual pediatric cases based on: history of trauma sustained in terms of days, infants age, dentition status, fracture site and degree of bony displacement, are all associated variables effecting treatment outcome for this procedure. However, successful outcome is determined by the effect that, early treatment is provided and growth has occurred normally without compromising form and function of jaws [6].

The treatment via this technique reported is aimed at, establishing early functional occlusion while limiting any potential impact on normal growth as, intervention ranges from: observation to open reduction with internal fixation depending on the fracture pattern, stage of dentition, available resources, skill-experience of operating surgeon and the skeletal age $[7,8]$. Opinion is divided in offering treatment of the growing mandible between internal fixation techniques via resorbable plates, closed reduction, intermaxillary fixation (IMF), and using acrylic splints $[9,10]$. Primary dentition offers very weak anchorage while, first permanent molars are adequate for circumandibular wiring via acrylic splints in primary or mixed dentition stage [9]. However, in absence of adequate primary teeth, immobilization with acrylic splint, lingual splint or related other known soft splint procedures, Prof. katpar's DUHS Splinting technique used can provide a good alternate to reduce fracture jaw in position in infants and small children in selected cases, however is not a replacement for other techniques used [10].

As treatment of fractured jaws is complex, time, high cost dependent and also needs competent surgical expertise, so the main objectives of treatment of mandible fracture via this splint technique reported are: avoid damage to existing deciduous dentition- prevent damage to tooth buds, avoid maxillomandibular fixation, avoid resorbable bone plates, reducing cost. In addition, Prof Katpar's technique does not need any specialized Skill and associated material but, only a decision to apply. At same time it promotes: early normal form- function and provides intra and extra oral dual stability of fractured jaw that supports healing, allowing normal eating, drinking with restoration of esthetics [11]. Followup for this case showed complete healing without any complications on the surrounding tissues, good alignment of existing single tooth with no occlusal disharmony or temporomandibular joint problems. We faced problems in taking all related pre and postoperative pictures for this case due to infantile age of the infant patient irritability. We recommend, associated problems with this technique should be anticipated by operating surgeons to prevent all related issues/complications.
The clinical outcomes indicate that immediate splints created from simple compound material, routinely used in Prosthodontics Specialty of Clinical Dentistry, can also be used for conservative treatment of infantile mandibular fracture as: immediate, costeffective, easy to use, devoid of impression taking, less timeconsuming, less general anesthesia and provides maximum stability during the healing period with minimal trauma to the adjacent anatomic structures [12]. However, periodic long-term follow-up is absolutely essential for the early determination of possible growth disturbances and we recommend that, this technique should only be used to treat pediatric population fracture mandible cases having fresh history of trauma not more than 4 days old. Via this technique, the Impression compound cake splint replaces routinely used Acrylic Splint and is secured via circummandibular silk thread, replacing Stainless Steel wire used for all such cases. It further avoids the need of costly resorbable bone plates and also avoids double general anesthesia used by some surgeons for making acrylic splints in pediatric population with further avoiding associated Prosthetic Lab Support. In addition, we have also treated few more pediatric fracture mandible cases using Prof. Katpar's DUHS Splinting technique with minor modifications, giving wonderful results.

\section{Conclusion}

By practicing "Prof. Katpar's DUHS splinting technique" being; efficient, cost effective, technically simple, causes less morbidity and allows immediate restoration of form and function in selective pediatric patients with most recent trauma history. We want to promote this as an, innovate, cost effective and safe new procedure with minimum mobility. This Surgical technique was also presented at Scientific Session at 11th PCOMS-Pakistan Conference of Oral and Maxillofacial Surgery, November 2018 at Rawalpindi, Pakistan and also at Anton Hipp Craniomaxillofacial Congress in July 2019 at Dresden/Germany.

\section{Author's Contributions}

Prof. Shahjahan Katpar: As corresponding Author, Pioneer for developing Concept/ Technique, operating and performing Prof. Katpar's splint technique on related patients and All remaining Author's supported in Case Preparation at variable stages and levels.

\section{Competing Interest}

The authors declare that they have no competing interest of conflict.

\section{Funding}

The authors declare that they have not received any funding for this technique. 
This Pioneer Surgical Technique is dedicated to my dearest father Mr Abdul Waheed Katpar for his encouraging teachings to "think out of the box" so that we can positively contribute in this world.

\section{Bibliography}

1. Nezam S., et al. "Management of mandibular fracture in pediatric patient". National Journal of Maxillofacial Surgery 9.1 (2018): 106-109.

2. Kumar N., et al. "Circummandibular wiring: A treatment approach toward management of mandibular fracture in children". International Journal of Clinical Dentistry and Research 1 (2017): 1-3.

3. Iida S and Matsuya T. "Paediatric maxillofacial fractures: Their aetiological characters and fracture patterns". Journal of Cranio-Maxillofacial Surgery 30.4 (2002): 237-241.

4. Gawelin PJ and Thor AL. "Conservative treatment of paediatric mandibular fracture by the use of orthodontic appliance and rubber elastics: Report of a case". Dental Traumatology 21.1 (2005): 57-59.

5. Haug RH and Foss J. "Maxillofacial injuries in the pediatric patient”. Oral Surgery, Oral Medicine, Oral Pathology, Oral Radiology, and Endodontics 90.2 (2000): 126-134.

6. Abdulaziz B., et al. "The Lingual Splint: An Often Forgotten Method for Fixating Pediatric Mandibular Fractures". Journal of the Canadian Dental Association 73.6 (2007): 521-524.

7. Goth S., et al. "Management of pediatric mandible fractures". Journal of Craniofacial Surgery 23.1 (2012): 47-56.

8. Sharma S., et al. "Pediatric mandibular fractures: A review". International Journal of Clinical Pediatric Dentistry 2.2 (2009): 1-5.

9. Crean ST., et al. "Conservative approach in the management of mandibular fractures in the early dentition phase. A case report and review of the literature". International Journal of Paediatric Dentistry 10.3 (2000): 229-233.

10. Emmanuel DS., et al. "Thermoformed splints in the management of pediatric mandibular fracture - A case report". SRM University Journal of. Dental Sciences 1 (2010): 240-242.
11. Katpar S., et al. "Maxillofacial trauma spectrum at Civil Hospital Karachi: A report from largest tertiary care Public sector teaching hospital in Sindh Province". Journal of Khyber College of Dentistry 5.2 (2015): 7-11.

12. Aggarwal H., et al. "Role of dental impression compound in plastic surgery: Know more about it". Indian Journal of Plastic Surgery 47.2 (2014): 273-275.

\section{Assets from publication with us}

- Prompt Acknowledgement after receiving the article

- Thorough Double blinded peer review

- Rapid Publication

- Issue of Publication Certificate

- High visibility of your Published work

Website: www.actascientific.com/

Submit Article: www.actascientific.com/submission.php

Email us: editor@actascientific.com

Contact us: +919182824667 